Research Paper

\title{
Expression of EMT-Related Genes CAMK2N1 and WNT5A is increased in Locally Invasive and Metastatic Prostate Cancer
}

\author{
Isa Carneiro ${ }^{1,2}$, Filipa Quintela-Vieira ${ }^{1,3}$, João Lobo ${ }^{1,2,5}$, Catarina Moreira-Barbosa ${ }^{1}$, Francisco Duarte

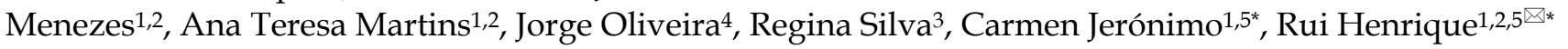 \\ 1. Cancer Biology and Epigenetics Group, Research Center of Portuguese Oncology Institute of Porto (GEBC CI-IPOP), R. Dr. António Bernardino de Almeida, \\ 4200-072, Porto, Portugal; \\ 2. Department of Pathology, Portuguese Oncology Institute of Porto (IPO Porto), R. Dr. António Bernardino de Almeida, 4200-072, Porto, Portugal \\ 3. School of Health, Polytechnic of Porto (ESS), R. Dr. António Bernardino de Almeida 400, 4200-072 Porto, Portugal; \\ 4. Department of Urology, Portuguese Oncology Institute of Porto (IPO Porto), R. Dr. António Bernardino de Almeida, 4200-072, Porto, Portugal; \\ 5. Department of Pathology and Molecular Immunology, Institute of Biomedical Sciences Abel Salazar, University of Porto (ICBAS-UP), Rua Jorge Viterbo \\ Ferreira 228, 4050-513, Porto, Portugal
}

*Joint senior authors

$\triangle$ Corresponding author: Rui Henrique, MD, PhD. ORCID: 0000-0003-3171-4666 Full mailing address: Department of Pathology, Portuguese Oncology Institute of Porto, Rua Dr. António Bernardino de Almeida, 4200-072, Porto, Portugal. Telephone number: 00351965659179 (mobile)/ 00351225084000 (work) Fax number: 00351225084199 E-mail address: rmhenrique@icbas.up.pt / henrique@ipoporto.min-saude.pt

(C) The author(s). This is an open access article distributed under the terms of the Creative Commons Attribution License (https://creativecommons.org/licenses/by/4.0/). See http://ivyspring.com/terms for full terms and conditions.

Received: 2019.03.03; Accepted: 2019.07.16; Published: 2019.10.15

\begin{abstract}
Purpose: Prostate cancer (PCa) varies clinically from very indolent, not requiring therapeutic intervention, to highly aggressive, entailing radical treatment. Currently, stratification of PCa aggressiveness is mostly based on Gleason score, serum PSA and TNM stage, but outcome prediction in an individual basis is suboptimal. Thus, perfecting pre-therapeutic discrimination between indolent and aggressive PCa, avoiding overtreatment is a major challenge. Epithelial to mesenchymal transition (EMT) allows epithelial cells to acquire mesenchymal properties, constituting a critical step in tumor invasion and metastization. Thus, we hypothesized that EMT-related markers might allow for improved assessment of PCa aggressiveness. Methods and Results: Using RealTime ready Custom Panel 384 assay, 93 EMT-related genes were assessed in normal prostate tissues (NPT, $n=5)$, stage $p T 2 a+b-P C a(n=5)$ and stage $\mathrm{pT} 3 \mathrm{~b}-\mathrm{PCa}(\mathrm{n}=5)$, from which CAMK2NI, CD44, KRTI4, TGF 33 and WNT5A genes emerged as the most significantly altered. Expression levels were then evaluated in a larger series (16 NPT and $94 \mathrm{PCa}$ ) of frozen tissues using quantitative RT-PCR. Globally, CAMK2NI, CD44 and WNT5A displayed higher expression levels at higher stages and less differentiated PCa. CAMK2N1 and WNT5A immunoexpression analysis disclosed significantly lower expression in NPT and increasing proportion of high-expression cases from $\mathrm{pT} 2 \mathrm{a}+\mathrm{b}$ to $\mathrm{pT} 3 \mathrm{~b}$ and metastatic PCa. Furthermore, higher CAMK2NI and WNT5A transcript levels associated with shorter disease-free and disease-specific survival. In multivariable analysis, a trend for WNT5A expression levels to independently predict DFS was disclosed $(p=0.056)$. Conclusions: Globally, our findings suggest an association between PCa aggressiveness and increased expression of CAMK2NI and WNT5A, reflecting the acquisition of effective EMT characteristics by PCa cells.
\end{abstract}

Key words: Prostate cancer, epithelial-to-mesenchymal transition, prognosis, CAMK2N1, WNT5A

\section{Introduction}

Prostate cancer (PCa) is the second most frequently diagnosed cancer and the fifth leading cause of cancer-related death among men worldwide, being the most frequently diagnosed male cancer in developed countries [1]. Currently, PCa management and treatment is decided based on serum 
prostate-specific antigen (PSA) levels, stage, histopathological characteristics and patient's life expectancy, among other factors [2]. However, PCa behavior is frequently unpredictable, ranging from very indolent, amenable to active surveillance, to highly aggressive, requiring radical treatment [3]. Whereas organ-confined PCa is mostly curable, locally or systemically advanced disease is uncurable as no effective curative treatments are available [2]. Due to the inability to accurately distinguish tumors that will be harmless from those that will be lethal in many cases, physicians and patients tend to elect treatments with curative intent, entailing undesirable side-effects without a clear survival benefit $[4],[5,6]$ $[5,7]$. Thus, improved characterization of tumor aggressiveness is critical for reducing both overtreatment and mortality and it should be ideally based on the biological properties of PCa cells.

Epithelial-to-mesenchymal transition (EMT) is a process that has been associated with tumor aggressiveness, playing a central role in tumor invasion and metastasis [8, 9]. EMT is a biologic process that allows epithelial cells to undergo multiple biochemical changes enabling the acquisition of a mesenchymal phenotype, thus enhancing migratory capacity, invasiveness, resistance to apoptosis, and augmenting the production of extracellular matrix components [10]. During this transition, epithelial cells progressively loose expression of typical epithelial markers, such as E-cadherin and keratins, and gain expression of typical mesenchymal markers, including vimentin, a-smooth muscle actin (SMA) and N-cadherin $[8,10]$. EMT endows cancer cells with mobility and the capacity to invade tissues and organs, as well as entering blood vessels [8]. After intravasation, these cancer cells are transported through the circulation and can leave the blood stream at a remote site where they may form metastasis [8]. Considering the important role of EMT in tumor progression and metastasis formation, EMT-related markers might be used to weigh tumor aggressiveness, allowing for improved PCa patient management. Hence, we sought to identify EMT-related genes involved in PCa progression, through evaluation of their expression levels in a series of PCa patients submitted to radical prostatectomy, and further assess their clinical usefulness for prediction of disease progression.

\section{Material and Methods}

\section{Clinical Samples}

PCa tissue samples were prospectively collected from patients with clinically localized disease, consecutively diagnosed and treated with radical prostatectomy at Portuguese Oncology Institute of Porto, Portugal (2001-2012). All patients were treated and followed-up by the same multidisciplinary team of this Institute. To allow for group comparisons, cases enrolled comprised either pT2a+b (representing early stage disease) or pT3b (representing locally advanced disease) PCa, encompassing a total of 94 tumor samples. Cases with insufficient clinical information were discarded from the analysis. Samples of morphologically normal prostate tissue (NPT, n=16) were collected from the peripheral zone of prostates obtained in the context of cystoprostatectomy performed in males with bladder cancer. Only specimens that did not harbor PCa nor prostatic intraepithelial neoplasia (PIN), confirmed after thorough sectioning and histological observation (and use of immunohistochemistry ancillary studies, whenever necessary), were included in the study and served as controls. Also, patients previously subjected to chemotherapy due to bladder cancer in the neoadjuvant setting were excluded.

All tissue samples were promptly frozen immediately after surgery, following patient informed consent, and stored at $-80^{\circ} \mathrm{C}$ for further analysis. After histological confirmation of $\mathrm{PCa}$ and NPT by a dedicated uropathologist in $5 \mu \mathrm{m}$-thick stained section, fresh-frozen tissue fragments were trimmed to maximize the yield $(>70 \%)$ of target cells. Subsequently, an average of fifty $12 \mu \mathrm{m}$ thick sections were cut and every fifth sections a $5 \mu \mathrm{m}$ section was stained to ensure a uniform percentage of target cells and to exclude contamination from neoplastic cells in normal tissue samples.

Secondly, formalin-fixed paraffin-embedded (FFPE) tissue samples from the same surgical specimens, collected for routine histopathological evaluation, were also used in this study. Regarding the macroscopic handling, prostate glands were totally embedded for histological examination and fragments were mapped according to the specific protocol of the Pathology Department. Given the greater availability of these FFPE samples compared to fresh frozen tissues, the series was further enriched in NPT samples (to the original 16 samples with fresh frozen tissue available already included, 29 FFPE samples were added, derived from further cystoprostatectomy specimens fulfilling the same requirements). Consultation cases were excluded. Gleason score [11], as well as pathological stage [12] of all PCa cases were assessed in histological slides from FFPE tissues fragments routinely collected from the same surgical specimens.

Thirdly, a set of metastatic PCa tissues (MET, $\mathrm{n}=57 ; 27$ bone metastases, 18 lymph node metastases and 12 metastases from other regions) were also 
selected for immunoexpression analysis. Cases without sufficient amount of tumor cells and/or artifacts (namely in bone biopsies) were excluded. Relevant clinical data was retrieved from clinical charts.

This study, as well as the use of samples and the access to clinical data, was approved by the institutional review board (Comissão de Ética para a Saúde) of Portuguese Oncology Institute of Porto, Portugal (CES-IPOFG_EPE 019/08).

\section{RNA Extraction}

Total RNA was extracted from fresh frozen samples using TRIzol method. Briefly, samples were suspended in TRIzol ${ }^{\circledR}$ reagent (Invitrogen) and, after homogenization using rotor-shaker, chloroform (Merck) was added. RNA was purified from the upper/aqueous phase of TRIzol extract using the PureLinkTM RNA Mini Kit (Invitrogen) according to the manufacturer's instructions. RNA concentration and purity ratios were then evaluated using NanoDrop ND-1000 spectrophotometer (ThermoScientific, Wilmington, DE, USA). Additionally, RNA integrity was checked by electrophoresis in $2 \%$ agarose-gel.

\section{Screening of 93 EMT Related Genes Expression}

Based on a comprehensive literature review, 93 potential EMT-related genes were selected for gene expression evaluation (Table S1). Five NPT and ten PCa samples representing the tumor spectrum in this series considering pathological stage $[\mathrm{pT} 2 \mathrm{a}+\mathrm{b}-\mathrm{PCa}$ $(n=5)$ and pT3b-PCa $(n=5)]$ were chosen for this analysis.

cDNA was synthesized from 1000ng of total RNA by reverse transcription using Transcriptor High Fidelity cDNA Synthesis Kit v.6 (Roche Applied Science, Mannhein, Germany), according to manufacturer's instructions.

Expression levels of 93 EMT-related genes were evaluated using RealTime ready Custom Panel 384 assay (Roche Applied Science, Mannhein, Germany). The QRT-PCR reaction was performed in a RealTime termocycler LightCycler ${ }^{\circledR} 480$ II (Roche Applied Science, Mannhein, Germany), following manufacturer's instructions. Each experiment was performed in triplicates for PCa samples and without replicates for NPT.

The amount of mRNA of the genes studied was normalized to that of TFRC reference gene and the median value of NPT, pT2a+b-PCa and pT3b-PCa samples was chosen to calculate fold-difference in gene expression among groups, using the comparative $\mathrm{Ct}$ method $\left(2^{-\Delta \Delta \mathrm{Ct})}\right)$. The $2^{-\Delta \Delta \mathrm{Ct}}$ values inferior to 0.5 were considered to indicate a significant expression reduction, whereas $2^{-\Delta \Delta C t}$ values above 2.0 indicated significant increase in expression. From the 93 EMT related genes analyzed, five genes were selected as potential markers of EMT in PCa based on differential expression between pT3b-PCa, NPT and pT2a+b-PCa, and correlation with gene behavior described in literature.

\section{Validation of Selected Genes}

To validate the selected genes, expression levels were evaluated in a larger series of NPT $(n=16)$ and PCa frozen tissue samples [pT2a+b-PCa $(n=48)$ and pT3b-PCa $(n=46)$.

cDNA was synthesized from 300ng of total RNA using TransPlex ${ }^{\circledR} \quad$ Whole Transcriptome Amplification (WTA) Kit (Sigma-Aldrich ${ }^{\circledR}$ ) according to manufacturer's protocol. WTA reaction products were purified using QIAquick PCR Purification Kit (QIAGEN), according to manufacturer's protocol.

mRNA levels were evaluated using TaqMan ${ }^{\circledR}$ Gene Expression assays (Applied Biosystems) for the selected genes and for the endogenous control GUS $\beta$. The QRT-PCR assay was performed in 96-well plates on an Applied Biosystems 7500 Real-time PCR system (Applied Biosystems), according to the recommended protocol. All samples were run in triplicate and two water blanks were added to each plate as negative controls. Serial dilutions of WTA-cDNA synthesized from prostate total RNA were analyzed as standards, allowing the construction of a standard curve for relative quantification and PCR efficiency assessment, for each plate. To determine the relative expression levels for each sample, mean quantity of each gene was normalized with mean quantity of endogenous control GUS $\beta$.

\section{Immunohistochemistry}

CAMK2N1 and WNT5A expression was assessed by immunohistochemistry in $3 \mu \mathrm{m}$ sections of FFPE samples [45 NPT, 94 PCa (pT2a+b and pT3b) and $57 \mathrm{MET}$. The technique was performed using anti-CAMK2N1 goat polyclonal antibody (Santa Cruz Biotechnology Inc.; sc-161427) at 1:75 dilution with ImmunoCruz goat $\mathrm{ABC}$ Staining System (Santa Cruz Biotechnology Inc.; sc-2023), and anti-WNT5A mouse monoclonal antibody (abcam; 3D10-ab86720) at 1:2500 dilution with Nonolink ${ }^{\mathrm{TM}}$ Max Polimer Detection System (Leica Biosystems). Antigen retrieval was performed by microwaving the sections at $800 \mathrm{~W}$ for 20-30 minutes in Citrate buffer, for both markers. Normal brain was used as positive control for CAMK2N1 and lung adenocarcinoma for WNT5A. Immunoexpression was scored as 1 (weak expression $\leq 50 \%$ of cells), 2 (weak expression $\geq 50 \%$ of cells or 
moderate expression $\leq 50 \%$ of cells) or 3 (moderate expression in $>50 \%$ of cells or intense expression, typically $>50 \%$ of cells).

A flow-chart of the sample selection, cohorts used and including and excluding criteria is presented in Supplementary Figure 1.

\section{In silico validation in an independent cohort}

To validate transcript and protein results

A

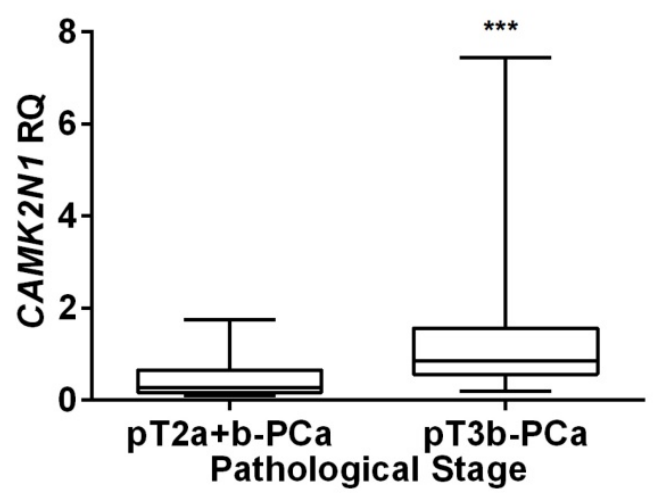

C

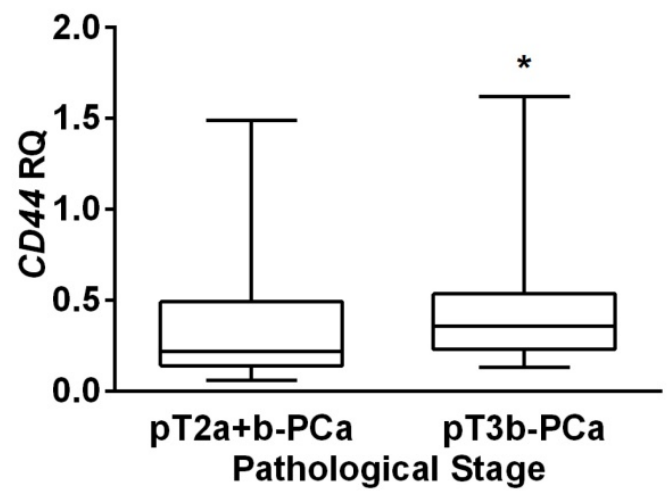

E

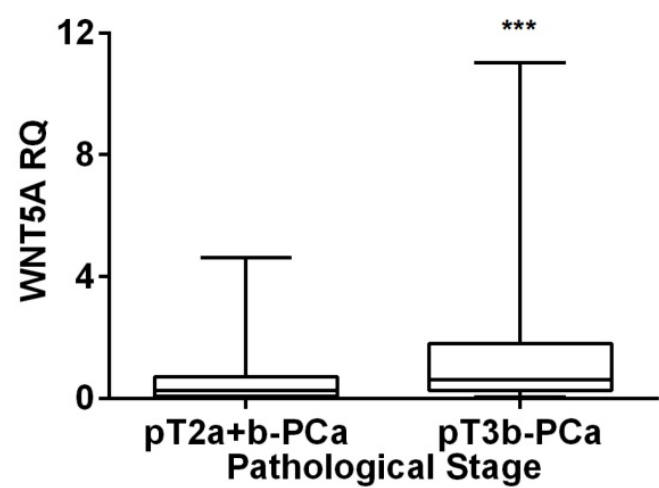

obtained in our own tissue cohort we performed an in silico analysis of the publicly available The Cancer Genome Atlas (TCGA) database, by using the online resource cBioPortal for Cancer Genomics [13]. mRNA data regarding both CAMK2N1 and WNT5A was imported from the online database, after which statistical analysis was performed.

B

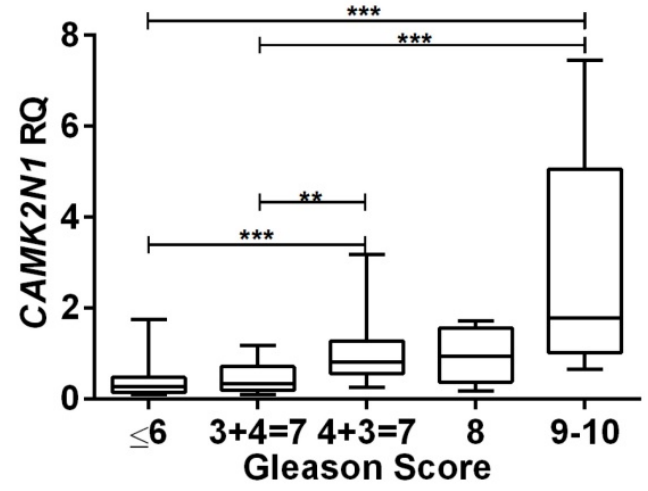

D

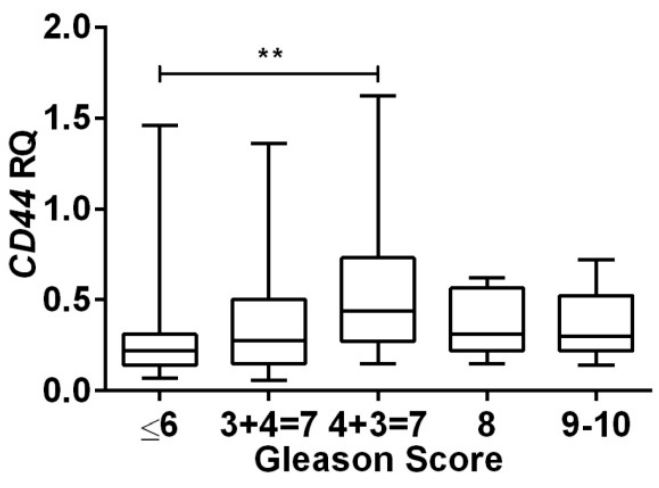

$\mathbf{F}$

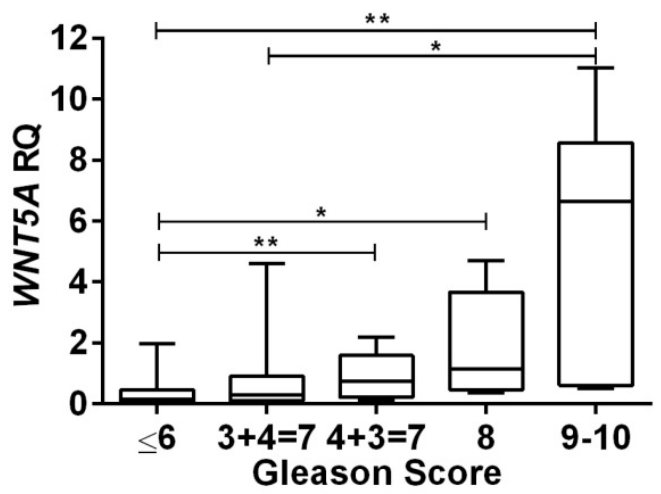

Figure 1 - Association of CAMK2NI, CD44 and WNT5A relative expression levels with clinicopathological data. Distribution of CAMK2NI(A), CD44(C) and WNT5A(E) expression levels according to pathological stage, showing higher expression levels in more advanced tumors, PT3b-PCa. Distribution of CAMK2NI(B), CD44(D) and WNT5A(F) expression levels according to Gleason Score, showing higher expression levels associated with a higher Gleason Score, mainly for CAMK2NI and WNT5A. 


\section{Statistical analysis}

Samples were divided into categories according to pTNM (NPT, pT2a+b-PCa and pT3b-PCa, MET) and GS (GS $\leq 6$; GS 3+4=7; GS 4+3=7; GS=8; GS=9-10).

The Shapiro-Wilk's W-test allowed for the examination of the appropriateness of a normal distribution assumption for each of the parameters (data not shown).

Differences in expression levels of the EMT-related genes between the different groups of samples were firstly analyzed using Kruskal-Wallis one-way ANOVA, followed by non-parametric Mann-Whitney U-test, when appropriate. A non-parametric Spearman test was performed to assess correlation between gene expression levels and serum PSA and age.

The prognostic significance of available clinical variables (pathological stage, GS, age and serum PSA levels) was assessed by constructing disease-specific (DSS) and disease-free survival (DFS) curves using Kaplan-Meier method with log-rank test (univariable analysis). A Cox-regression model comprising the four variables (multivariable analysis) was also constructed. DFS was calculated from the date of the radical prostatectomy to the date of biochemical relapse, or date of last follow up, or death if relapse-free. For the purposes of survival analyses, all cases were coded based on the expression levels of each gene using percentile 75 value as empirical threshold. Cases were also subdivided according to serum PSA levels (below and above median values) and age (above 60, between 60 and 70, and above 70 years). Immunoexpression within groups of samples was compared using the Chi-square test, and to measure the strength and direction of associations, Somers' D test was additionally performed.

For the in silico analysis samples were grouped as close as possible to the categories already analyzed in the tissue cohort: pT2 vs pT3b tumors; GS $=6$ vs GS $=7$ vs $G S=8$ vs $G S \geq 9$; and additionally the comparison between $\mathrm{pN} 0$ and $\mathrm{pN} 1$ cases. Distribution of continuous variables between groups was also compared using the nonparametric Mann-Whitney U-test. Survival curves were also plotted using the Kaplan-Meier method and log rank test was used for survival analysis.

The level of significance was set at $p<0.05$. For multiple comparisons, Bonferroni's correction and Dunn's test were used to adjust p-values. Statistical analysis was performed using IBM ${ }^{\circledR}$ SPSS $®$ Statistics 23 software and GraphPad Prism 6.

\section{Results}

\section{Clinical Samples}

For the purposes of this study, NPT and PCa tissue samples were used to evaluate transcript levels and protein expression of EMT-related genes. Relevant clinical and pathological data are summarized in Table 1. No significant differences in age distribution between NPT and PCa groups were found.

\section{Expression of EMT-related genes and clinicopathological correlates}

Expression levels of 93 EMT-related genes were evaluated in five NPT and ten PCa samples. According to the previously described criteria, CAMK2N1, CD44, KRT14, TGFB3 and WNT5A were selected for validation. Array results disclosed decreased expression of CD44, KRT14 and TGFB3 in PCa-pT3b compared to PCa-pT2a+b, whereas the opposite was depicted for WNT5A and CAMK2N1 (Table 2).

Expression levels of the selected five EMT-related genes were then assessed in a larger series of 15 NPT and 94 PCa frozen tissue samples. Comparing $\mathrm{PCa}$ to NPT, statistically significant differences were confirmed for CD44 ( $p=0.013)$, KRT14 ( $\mathrm{p}=0.004)$ and WNT5A $(\mathrm{p}<0.001)$, but not for CAMK2N1 and TGFB3.

Table 1. Clinical and pathological features of patients included in this study.

\begin{tabular}{|c|c|c|c|c|c|c|c|}
\hline & \multicolumn{2}{|l|}{ Cohort 1} & \multicolumn{2}{|l|}{ Cohort 2} & \multicolumn{3}{|l|}{ Cohort 3} \\
\hline & NPT & $\mathrm{PCa}$ & NPT & $\mathrm{PCa}$ & NPT & $\mathrm{PCa}$ & Met \\
\hline Patients, $n$ & 5 & 10 & 16 & 94 & 45 & 94 & 57 \\
\hline Median Age, yrs (range) & $61(54-66)$ & $61(53-74)$ & $63(45-80)$ & $63(50-75)$ & $66(45-82)$ & $63(50-75)$ & \\
\hline PSA ( $\mathrm{ng} / \mathrm{mL})$, median (range) & n.a. & $7.75(4.5-19.9)$ & n.a. & $9.75(2.0-25.4)$ & n.a. & $9.75(2.0-25.4)$ & n.a \\
\hline \multicolumn{8}{|l|}{ Pathological Stage, $n(\%)$} \\
\hline pT2a or pT2b & n.a. & $5(50.0)$ & n.a. & $48(51.1)$ & n.a. & $48(51.1)$ & n.a \\
\hline pT3b & n.a. & $5(50.0)$ & n.a. & $46(48.9)$ & n.a. & $46(48.9)$ & n.a \\
\hline \multicolumn{8}{|l|}{ Gleason Score, $n(\%)$} \\
\hline$\leq 6$ & n.a. & $1(10.0)$ & n.a. & $27(28.7)$ & n.a. & $27(28.7)$ & n.a \\
\hline $3+4=7$ & n.a. & $4(40.0)$ & n.a. & $26(27.7)$ & n.a. & $26(27.7)$ & n.a \\
\hline $4+3=7$ & n.a. & $0(0.0)$ & n.a. & $27(28.7)$ & n.a. & $27(28.7)$ & n.a \\
\hline 8 & n.a. & $0(0.0)$ & n.a. & $5(5.3)$ & n.a. & $5(5.3)$ & n.a \\
\hline $9-10$ & n.a. & $5(50.0)$ & n.a. & $9(9.6)$ & n.a. & $9(9.6)$ & n.a \\
\hline
\end{tabular}

NPT, normal prostatic tissue; PCa, prostate cancer; Met, metastatsis; n.a., not available/applicable. 
Table 2. Values of $2^{-{ }^{\Delta}} \mathrm{Ct}$ between $\mathrm{PT} 3 \mathrm{~b}-\mathrm{PCa}$ and NPT, and PT2a+b-PCa and pT3b-PCa for selected genes.

\begin{tabular}{lll}
\hline & $2-\Delta \Delta$ Ct & \\
\cline { 2 - 3 } & pT3b-PCa Vs. NPT & pT3b-PCa Vs. pT2a+b-PCa \\
\hline CD44 & 0.16 & 0.31 \\
KRT14 & 0.19 & 0.28 \\
TGFB3 & 0.21 & 0.26 \\
WNT5A & 2.68 & 9.16 \\
CAMK2N1 & 4.76 & 4.04
\end{tabular}

NPT, normal prostatic tissue; pT3b-PCa, prostate cancer pathological stage pT3b; pT2a+b-PCa, prostate cancer pathological stage pT2a and pT3b.

A significant correlation between CAMK2N1 expression levels, but not CD44, KRT14, TGFB3 and WNT5A, and serum PSA levels of PCa patients was found $(\mathrm{r}=0.221, \mathrm{p}=0.034)$.

Concerning pathological stage, CAMK2N1 $(\mathrm{p}<0.001)$, CD44 $(\mathrm{p}=0.020)$ and WNT5A $(\mathrm{p}<0.001)$ expression levels were significantly higher in locally advanced (pT3b-PCa) compared to organ-confined (pT2a+b-PCa) disease (Figure 1A, C and E), but no significant differences for KRT14 and TGFB3 were depicted.

Analysis of variance indicated significant differences in expression levels among the five GS categories for CAMK2N1 ( $\mathrm{p}<0.001), C D 44(\mathrm{p}=0.015)$ and WNT5A ( $\mathrm{p}<0.001)$. Globally, highest expression levels were found in higher GS categories and

A

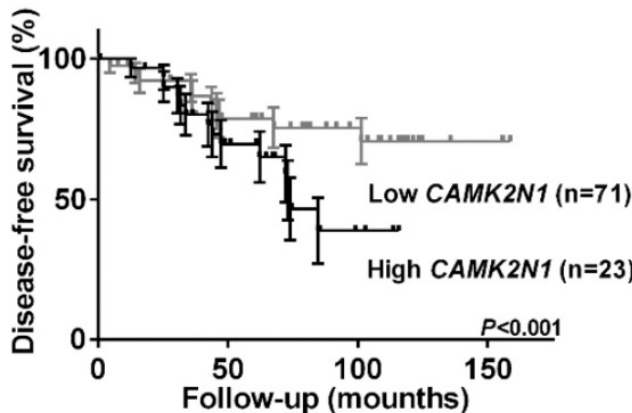

C

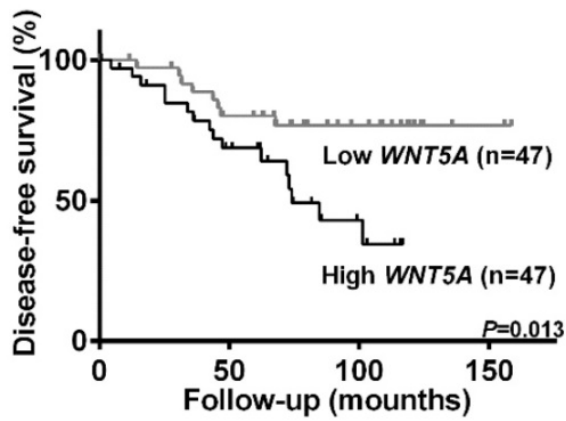

pair-wise comparisons, using Mann-Whitney U-test, CAMK2N1 and WNT5A revealed significant differences among GS categories (Figure 1B, D and F).

\section{Survival analyses}

The median follows up period of this series of primary PCa patients was 91 months (range: 3-161 months). At the time of the last follow-up, six patients $(6.4 \%)$ had died from PCa and 24 (25.5\%) developed biochemical recurrence. In 17 patients, serum PSA levels $>0.2 \mathrm{ng} / \mathrm{mL}$ persisted following surgery and these were not further considered for disease-free survival (DFS) analysis. As expected, pT3b PCa patients experienced significantly reduced DFS compared to $\mathrm{pT} 2 \mathrm{a}+\mathrm{b}$ PCa $(\mathrm{p}=0.032)$, and $\mathrm{GS}=8$ / $\mathrm{GS}=9-10 \mathrm{PCa}$ also associated with shorter time to relapse compared to the remainder categories $(p<0.001)$. The same trend was observed for DSS [higher pathological stage $(\mathrm{p}=0.02)$ and GS category $(\mathrm{p}<0.001)$ associated with decreased DSS].

Higher CAMK2N1 (Figure 2A and 2C) and WNT5A (Figure 2B and 2D) expression levels associated with shorter DFS $(p=0.045$ and $p=0.013$, respectively) and DSS ( $\mathrm{p}<0.001$ and $\mathrm{p}=0.003$, respectively). No significant associations were found for $C D 44$.
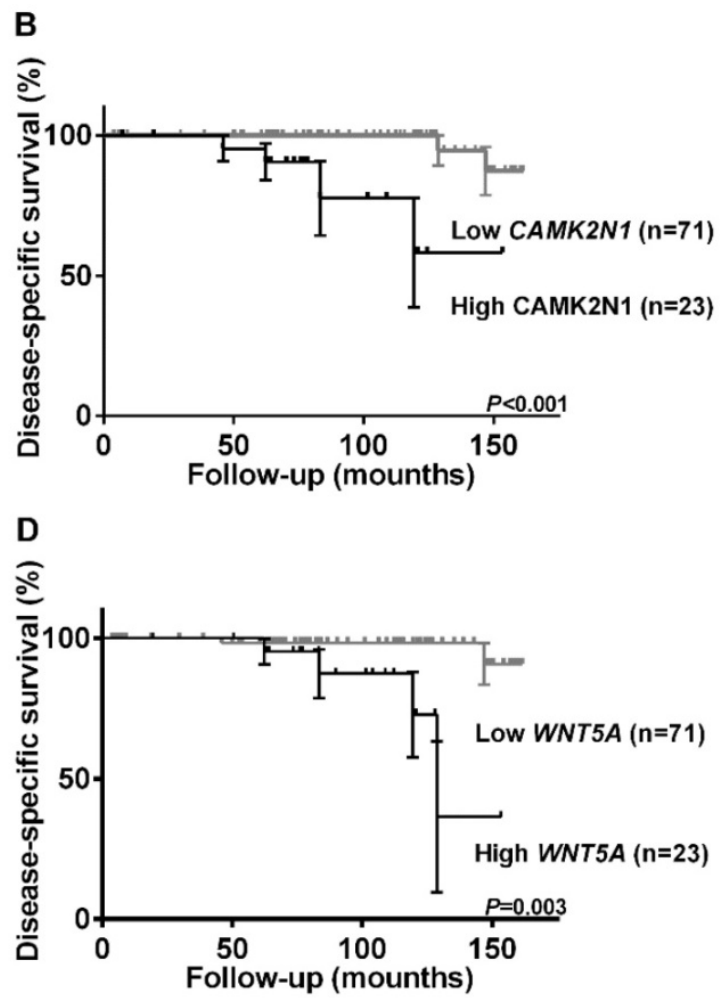

Figure 2 - Kaplan-Meier estimated disease-free survival and disease-specific survival curves of 94 PCa patients. Disease-free survival curves according to relative expression levels of CAMK2NI(A) and WNT5A (C); Expression levels were categorized using third quartile ( $75^{\text {th }}$ percentile) value as the cutoff. Disease-specific survival curves according to relative expression levels of CAMK2NI(B) and WNT5A (D); Expression levels were categorized using second quartile (50th percentile) value as the cutoff. 


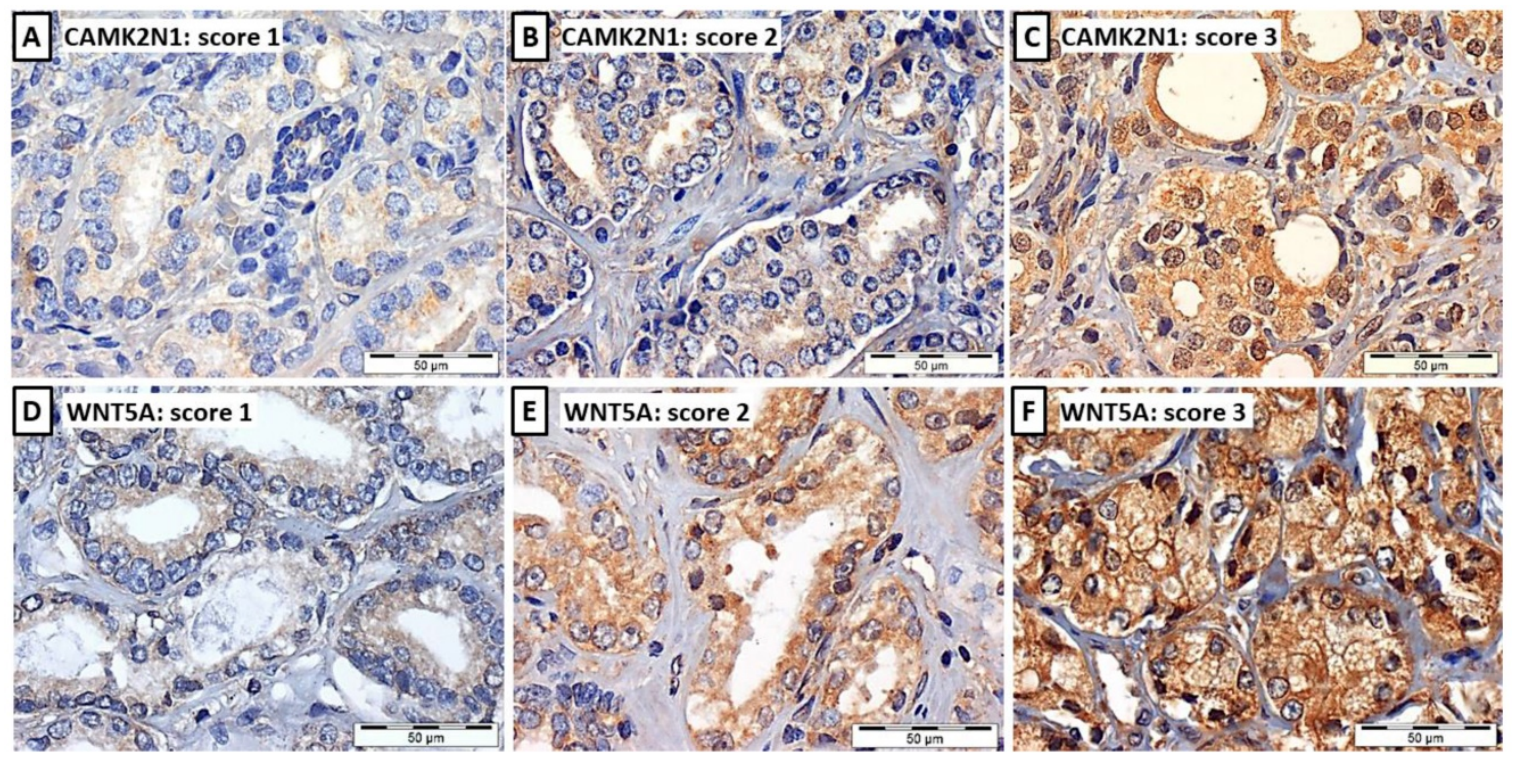

Figure 3 - Illustrative images of CAMK2N1 (A, B and C) and WNT5A (D, E and F) immunoexpression in PCa tissues, considering the 3-tiered immunoscore: immunoscore 1 (A and $\mathbf{D}$ ), weak expression $\leq 50 \%$ of cells, immunoscore 2 (B and $\mathbf{E}$ ), weak expression $\geq 50 \%$ of cells or moderate expression $\leq 50 \%$ of cells, and immunoscore 3 ( $\mathbf{C}$ and $\mathbf{F}$ ), moderate expression in $>50 \%$ of cells or intense expression, typically $>50 \%$ of cells.
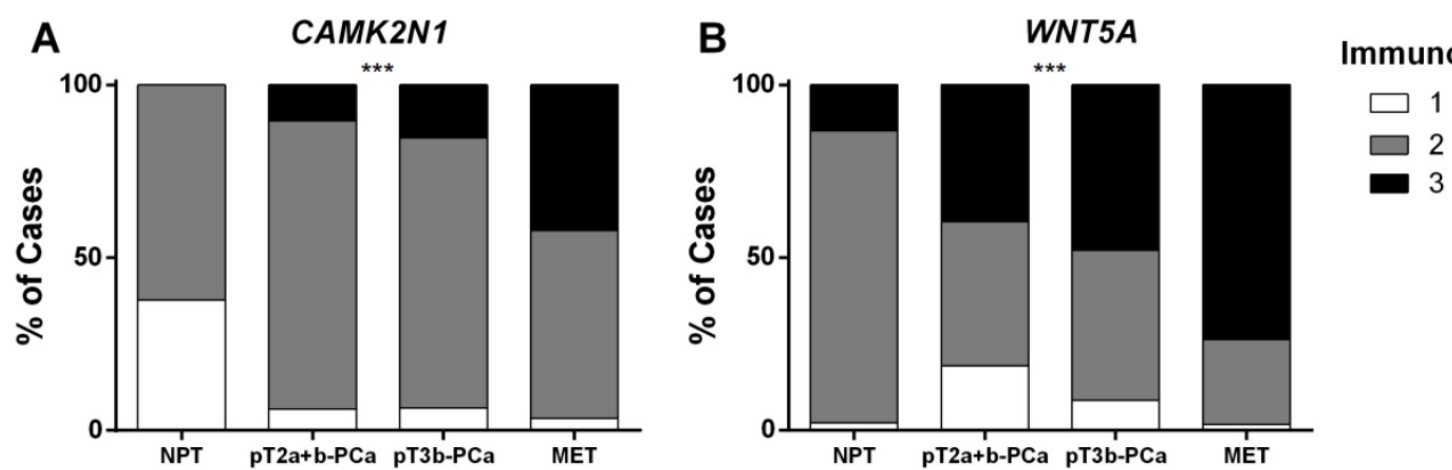

Figure 4 - Immunoexpression of CAMK2N1 and WNT5A in prostate tissue samples (Cohort\#3). Distribution among Cohort\#3 groups of (A) CAMK2N1 immunoexpression and (B) WNT5A immunoexpression.

In multivariable analysis, CAMK2N1 and WNT5A did not disclose independent prognostic value, although a trend for WNT5A expression levels independently predict DFS was disclosed $(\mathrm{p}=0.056)$.

\section{CAMK2N1 and WNT5A immunoexpression in primary and metastatic PCa}

CAMK2N1 and WNT5A immunoexpression assessment was carried out in $45 \mathrm{NPT}, 94 \mathrm{PCa}$ (pT2a+b and pT3b; Figure 3) and 57 PCa metastases (MET). These two genes were selected owing to the correlation found with pathological features and survival. Somers' D test disclosed significant differences in expression for both genes (Figure 4A and $4 \mathrm{~B})(\mathrm{p}<0.001$, for both). Lowest expression was displayed by NPT whereas an increase in the proportion of cases with higher expression was apparent from PCa-pT2a+b to PCa-pT3b and MET, for both CAMK2N1 and WNT5A (CAMK2N1: $\mathrm{v}=0.418, \mathrm{p}<0.001 ;$ WNT5A: $\mathrm{v}=0.347, \mathrm{p}<0.001)$.

A similar trend was apparent for WNT5A, but not CAMK2N1, immunoexpression according to GS category, with higher categories depicting higher proportion of cases with high immunoexpression (Somers' D: v=0.227, $\mathrm{p}=0.013$ ).

Survival analyses, however, did not disclose significant associations between CAMK2N1 or WNT5A immunoexpression and prognosis.

\section{In silico validation in the TCGA database}

A complete description of the PCa TCGA cohort can be found at [13]. Briefly, it includes 498 patients, with a median age at diagnosis of PCa of 61 years. In this series, like in ours, both CAMK2N1 $(p<0.0001)$ and WNT5A $(\mathrm{p}<0.001)$ are significantly upregulated in $\mathrm{pT} 3 \mathrm{~b}$ cases, when compared to pT2 tumors (Figure $5 \mathrm{~A}$ and 5D). Also, again reproducing results in our cohort, higher Gleason score cases showed significantly higher expression of these players when compared to lower Gleason score tumors (Figure 5C and $5 \mathrm{~F}$ ). 

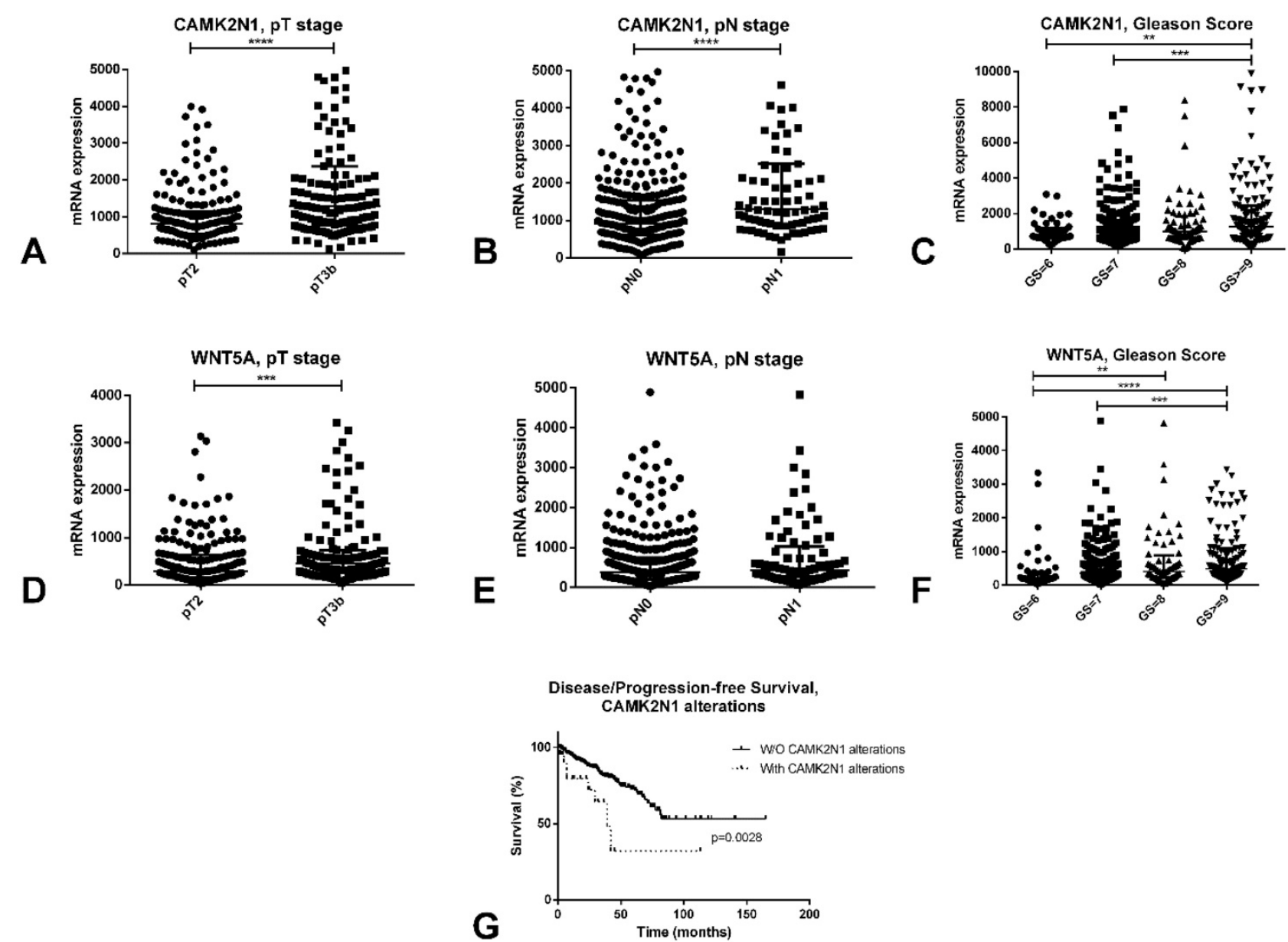

Figure 5 - In silico analysis of the TCGA database concerning CAMK2N1 and WNT5A. A-C: Differential mRNA expression of CAMK2N1 according to pT stage (A), pN stage (B) and Gleason Score (C). D-F: Differential mRNA expression of WNT5A according to pT stage (D), pN stage (E) and Gleason Score (F). G - Disease/progression-free survival of prostate cancer patients according to CAMK2NI alterations.

Additionally, CAMK2N1 was found to be significantly upregulated in pN1 tumors when compared to pN0 $(\mathrm{p}<0.0001)$, although this was not observed for WNT5A (Figure 5B and 5E). Patients with alterations in CAMK2N1 also displayed significantly worse disease/progression-free survival when compared to those without deregulation of this gene $(p=0.0028$, Figure $5 G)$.

\section{Discussion}

Increasing the accuracy of predicting the potential clinical aggressiveness of $\mathrm{PCa}$ is a major challenge as it constitutes the mainstay to improve patient management and avoid overtreatment. Currently available tools for risk stratification of PCa patients, which include diagnostic serum PSA levels, clinical stage (cTNM) and Gleason score in biopsy [5, 6], are helpful but limited in their ability to accurately predict outcome or tumor aggressiveness [5, 7]. Because EMT plays an important role in tumor progression, fostering invasion and metastization [8], we hypothesized that quantitative evaluation of transcript levels of EMT-related genes might allow for improved stratification of PCa patients according to the likelihood of progression to lethal forms of disease.

Thus, we firstly assessed the expression levels of 93 EMT-related genes using a RealTime ready Custom Panel 384 assay, using a limited, though representative, number of $\mathrm{PCa}$ tissue samples, compared to normal prostate tissue. Selected tumor samples represented two distinct phases of $\mathrm{PCa}$ progression, i.e., small volume, organ-confined disease $(\mathrm{pT} 2 \mathrm{a}+2 \mathrm{~b})$, usually associated with good prognosis, and locally advanced disease ( $\mathrm{pT} 3 \mathrm{~b}$ ) which entails high risk of metastization. Although this approach might underestimate alterations in gene expression, it allows for selection of the most significantly altered genes. From array analysis, five candidate genes emerged (CAMK2N1, CD44, KRT14, TGF $\beta 3$ and WNT5A) and validation in a larger sample set was mandatory to sanction these preliminary findings. Interestingly, significant differences in gene expression between NPT and PCa were confirmed for 
CD44, KRT14 and WNT5A, but not for CAMK2N1 and TGF $\beta 3$. The results observed for CAMK2N1 might be explained by the low expression demonstrated by pT $2 a+b$ tumors, which is very similar to NPT, negatively impacting in the comparison PCa vs. NPT. On the other hand, the opposing results concerning CD44 expression in the array and in the validation series, might derive from the small number of cases analysed in the array and further emphasize the need for validation of array results.

Remarkably, statistically significant associations with pathological stage and Gleason score were disclosed for CAMK2N1, CD44 and WNT5A, with more advanced stage and higher GS tumors depicting higher transcript levels. Furthermore, similar results for CAMK2N1 and WNT5A expression were disclosed at protein level, evaluated through immunohistochemistry. Globally, these results indicate that increased expression of those EMT-related genes occurs along tumor progression, which is further underscored by the finding of increased CAMK2N1 and WNT5A immunoexpression in metastatic PCa. Since EMT endows tumor cells with the ability to invade, migrate and metastasize, these findings [8] seem to confirm the hypothesis which set the basis of our study. Interestingly, higher CAMK2N1 and WNT5A transcript levels associated with worse DFS and DSS, although only in univariable analysis. The relatively limited sample size might have impaired the confirmation of an independent prognostic value for both CAMK2N1 and WNT5A transcript levels, but the association with pathological stage and GS, two well established PCa prognostic parameters, is likely also to contribute to this result. Intriguingly, no prognostic value was disclosed for CAMK2N1 or WNT5A immunoexpression, but it should be recalled that immunoexpression analysis is a more subjective and error-prone evaluation than quantitative RT-PCR, which may not be able to capture subtle alterations in gene expression. Moreover, we performed an in silico analysis of an independent cohort (as already performed by us $[14,15])$, which validated our results. Indeed, upregulation of the two most remarkable players associated with $\mathrm{pT} 3 \mathrm{~b}$ tumors and higher Gleason scores. Additionally, overexpression of CAMK2N1 also associated with pN1 disease, putting in evidence its effect on PCa metastization, and alterations in this gene predicted poorer disease/progression-free survival.

CAMK2N1, CD44 and WNT5A have been previously associated with EMT in PCa and other malignancies. CD44, a transmembrane glycoprotein involved in cell adhesion, migration, differentiation, signal transduction and apoptosis [16, 17], has been proposed as a cancer stem cell (CSC) marker in several tumors [18-20], including PCa [21], in which also promotes cell migrations and invasion in vitro as well as metastatic dissemination in vivo and chemoresistance [21-23]. Recently, a CD44+ stem like cell was considered an initiator of EMT contributing to PCa metastasis, suggesting CD44 as poor prognosis marker in PCa [24]. Our results are in line with those findings since higher expression levels were found in more advanced and less differentiated tumors.

As for CAMK2N1, it encodes for a protein that interacts and inhibits CAMK2 signaling, which is implicated in cell cycle progression through activation of MEK/ERK and Notch-1 pathways [25, 26], both of which have been shown to induce EMT [27]. In PCa cell lines, CAMK2 overexpression induced a decrease in apoptosis, whereas its inhibition reduced proliferation and invasion capacity [28]. Thus, considering the actions mediated by CAMK2, a tumor suppressor role would be expected for CAMK2N1. Indeed, there is evidence that CAMK2N1 deactivates MEK/ERK pathway reducing tumor growth [29] and a tumor suppressive role in PCa has been proposed [30, 31]. Nevertheless, our findings suggest an oncogenic role for CAMK2N1 because higher expression was detected in $\mathrm{PCa}$, with significantly increased expression in more advanced tumors and metastases, and association with worse outcome. Survival analysis also demonstrated an association of an increased expression with a poor prognosis. Interestingly, increased CAMK2N1 expression was found in PCa cases that recurred [32]. A possible explanation for these finding is that tumor cells actively engaged in EMT are usually less proliferative [33]. Thus, the inhibitory activity of CAMK2N1 on CAMK2 in PCa would provide conditions for cancer cells to endure EMT through slowing cellular proliferative activity. However, the mechanism through which CAMK2N1 promotes tumor aggressiveness remains unclear and require functional studies.

WNT5A, a member of Wnt family, has been implicated in tumor progression and osteomimicky (a process in which prostate cancer cells acquire an osteoblast-like phenotype) [34]. Thus, it is expected to play an important role in $\mathrm{PCa}$ metastization considering the well-known propensity to metastasize to bone. Wnts signaling pathways are generally classified into canonical/ $\beta$-catenin dependent pathway and non-canonical/ $\beta$-catenin independent pathway. WNT5A is one of the most studied non-canonical Wnts, although it has been suggested that, in specific contexts, it may also interact with the canonical pathway [35, 36]. Intriguingly, although WNT5A has been generally found to be upregulated 
in $\mathrm{PCa}$, its role is still unclear, as some studies report association with good prognosis [37, 38] whereas other report associations with tumor aggressiveness and poor prognosis [39-41]. Indeed, more recent works have shown an implication of WNT5A in maintaining PCa cells dormancy in bone [42] and also that lower expression of this player and its receptors FZD5 and RYK associate with improved DSS [43]; however, it has also been shown that WNT5A induces castration resistant phenotype in the bone niche by interacting with macrophages [44] and that the protein SHISA2 interacts with WNT5A in mediating PCa aggressiveness [45]. Our findings support the latter hypothesis since we demonstrated WNT5A upregulation in $\mathrm{PCa}$, both at transcript and protein levels, associating with higher pathological stage and GS, metastization and worse prognosis.

The relatively small sample size and, to a lesser extent, the follow-up time constitute the main limitations of this study. As previously mentioned, this has most probably impacted negatively in multivariable analysis, precluding the confirmation of CAMK2N1 and WNT5A transcript levels as independent prognostic markers (despite a borderline result for WNT5A and DFS). Moreover, the lack of prognostic significance for CAMK2N1 and WNT5A immunoexpression is also limitative as immunohistochemistry is widely available in Pathology labs, facilitating translation of these findings into routine practice. Nevertheless, our study brought novelty (including methodological novelty) into the study of EMT-related genes in PCa, since it stemmed from a wide, array-based screen of several genes (selected after careful and thorough literature review) in a limited amount of contrasting samples (both normal parenchyma of non-neoplastic prostate glands and PCa samples with distinct aggressiveness), followed by validation in a much larger patient-derived cohort, treated by the same multidisciplinary team of the same institution and, importantly, subjected to the same rigorous pathological assessment. Moreover, besides validation of findings at the transcript level (including survival analyses), the study conclusions were further validated at the protein levels by immunohistochemistry, incrementing their value. Also, inclusion of metastatic samples provided a proof of concept for the study, allowing inferring on the effect of these players on the EMT and metastization phenomenon. Finally, and importantly, main conclusions were further validated in an independent, large and rigorous cohort, the TCGA database, which further strengthens the results.

In conclusion, we demonstrated that two EMT-related genes, CAMK2N1 and WNT5A, are potential biomarkers for assisting in assessment of PCa clinical aggressiveness. Indeed, increased expression was observed in more advanced and less differentiated tumors, as well as in metastases, associating with worse prognosis. These results require further validation in a more extended series of PCa cases, eventually including prostate biopsies to ascertain the ability to convey prognostic information in pre-treatment setting. Nevertheless, validations of the results in an independent cohort (TCGA) further strengthen our conclusions. However, we acknowledge that functional studies might help to further clarify the role of CAMK2N1 and WNT5A in PCa progression.

\section{Abbreviations}

CAMK2N1: Calcium/Calmodulin Dependent Protein Kinase II Inhibitor 1; CD44: Cluster of differentiation 44; DFS: Disease-free survival; DSS: Disease-specific survival; EMT: Epithelial-tomesenchymal transition; FFPE: Formalin fixed paraffin embedded; GS: Gleason score; KRT14: Keratin 14; IHC: Immunohistochemistry; MET: Metastatic prostate cancer tissue; NPT: Normal prostate tissue; PCa: Prostate cancer; PSA: Prostate specific antigen; QRT-PCR: Quantitative RT-PCR; SMA: a-smooth muscle actin; TGF $\beta 3$ : Transforming growth factor, beta 3; TNM: Tumor Node Metastasis staging system; WNT5A: Wingless-type MMTV integration site family, member $5 \mathrm{~A}$.

\section{Supplementary Material}

Supplementary figures and tables. http://www.jcancer.org/v10p5915s1.pdf

\section{Acknowledgements}

The authors are grateful to the patients that volunteered to provide samples and to all the personnel of the Department of Urology, Portuguese Oncology Institute - Porto that kindly collaborated in this study.

\section{Funding}

This study was funded by Research Centre of Portuguese Oncology Institute of Porto (CI-IPOP-17-2015). JL is supported by an FCT (Fundação para a Ciência e Tecnologia) fellowship (grant number SFRH/BD/132751/2017).

\section{Ethical approval}

All procedures performed in studies involving human participants were in accordance with the ethical standards of the institutional and/or national research committee and with the 1964 Helsinki declaration and its later amendments or comparable 
ethical standards.

\section{Informed consent}

Informed consent was obtained from all individual participants included in the study.

\section{Authors' contributions}

$\mathrm{CJ}$ and $\mathrm{RH}$ designed the study; IC, FQV, CM-B and ATM performed the molecular analyses and immunohistochemistry; $\mathrm{RH}$ and JL collected tissue samples, supervised tissue selection and evaluated immunohistochemistry; FDM and JO enrolled patients and collected clinical data; IC drafted the manuscript; RAS, CJ and RH revised the manuscript; all authors read and approved the final version of the manuscript.

\section{Competing Interests}

The authors have declared that no competing interest exists.

\section{References}

1. Torre LA, Bray F, Siegel RL, Ferlay J, Lortet-Tieulent J, Jemal A. Global cancer statistics, 2012. CA Cancer J Clin. 2015; 65: 87-108.

2. Damber JE, Aus G. Prostate cancer. Lancet. 2008; 371: 1710-21.

3. Moyer VA. Screening for Prostate Cancer: U.S. Preventive Services Task Force Recommendation Statement. Annals of internal medicine. 2012; 157: 120-34.

4. Ganz PA, Barry JM, Burke W, Col NF, Corso PS, Dodson E, et al. National Institutes of Health State-of-the-Science Conference: role of active surveillance in the management of men with localized prostate cancer. Annals of internal medicine. 2012; 156: 591-5.

5. Bégin LR, Bismar A.T. Prostate Cancer: A Pathological Perspective. In: Foulkes WDaC, editor. Male Reproductive Cancers: Epidemiology, Pathology and Genetics. New York: Springer; 2010.

6. Bastian PJ, Mangold LA, Epstein JI, Partin AW. Characteristics of insignificant clinical T1c prostate tumors. A contemporary analysis. Cancer. 2004; 101: 2001-5.

7. Ploussard G, Epstein JI, Montironi R, Carroll PR, Wirth M, Grimm MO, et al. The contemporary concept of significant versus insignificant prostate cancer. European urology. 2011; 60: 291-303.

8. Kalluri R, Weinberg RA. The basics of epithelial-mesenchymal transition. The Journal of clinical investigation. 2009; 119: 1420-8.

9. Thiery JP. Epithelial-mesenchymal transitions in tumour progression. Nature reviews Cancer. 2002; 2: 442-54

10. Kalluri R, Neilson EG. Epithelial-mesenchymal transition and its implications for fibrosis. The Journal of clinical investigation. 2003; 112: 1776-84.

11. Gleason DF, Mellinger GT. Prediction of prognosis for prostatic adenocarcinoma by combined histological grading and clinical staging. J Urol. 1974; 111: 58-64.

12. Sobin L.H. WCH. TNM classification of malignant tumours 6 th edition. New York: Wiley-Liss; 2002

13. Cerami E, Gao J, Dogrusoz U, Gross BE, Sumer SO, Aksoy BA, et al. The cBio cancer genomics portal: an open platform for exploring multidimensional cancer genomics data. Cancer Discov. 2012; 2: 401-4.

14. Lobo J, Henrique R, Jeronimo $C$. The Role of DNA/Histone Modifying Enzymes and Chromatin Remodeling Complexes in Testicular Germ Cell Tumors. Cancers (Basel). 2018; 11.

15. Lobo J, Barros-Silva D, Henrique R, Jeronimo C. The Emerging Role of Epitranscriptomics in Cancer: Focus on Urological Tumors. Genes (Basel). 2018; 9 .

16. Naor D, Sionov RV, Ish-Shalom D. CD44: structure, function, and association with the malignant process. Advances in cancer research. 1997: 71:241-319.

17. Ponta H, Sherman L, Herrlich PA. CD44: from adhesion molecules to signalling regulators. Nature reviews Molecular cell biology. 2003; 4: 33-45.

18. Al-Hajj M, Wicha MS, Benito-Hernandez A, Morrison SJ, Clarke MF Prospective identification of tumorigenic breast cancer cells. Proceedings of the National Academy of Sciences of the United States of America. 2003; 100: 3983-8.

19. Prince ME, Sivanandan R, Kaczorowski A, Wolf GT, Kaplan MJ, Dalerba P, et al. Identification of a subpopulation of cells with cancer stem cell properties in head and neck squamous cell carcinoma. Proceedings of the National Academy of Sciences of the United States of America. 2007; 104: 973-8.
20. Li C, Heidt DG, Dalerba P, Burant CF, Zhang L, Adsay V, et al. Identification of pancreatic cancer stem cells. Cancer research. 2007; 67: 1030-7.

21. Patrawala L, Calhoun T, Schneider-Broussard R, Li H, Bhatia B, Tang S, et al. Highly purified CD44+ prostate cancer cells from xenograft human tumors are enriched in tumorigenic and metastatic progenitor cells. Oncogene. 2006; 25: 1696-708.

22. Desai B, Rogers MJ, Chellaiah MA. Mechanisms of osteopontin and CD44 as metastatic principles in prostate cancer cells. Molecular cancer. 2007; 6: 18.

23. Hao J, Madigan MC, Khatri A, Power CA, Hung TT, Beretov J, et al. In Vitro and In Vivo Prostate Cancer Metastasis and Chemoresistance Can Be Modulated by Expression of either CD44 or CD147. PloS one. 2012; 7: e40716.

24. Shang Z, Cai Q, Zhang M, Zhu S, Ma Y, Sun L, et al. A switch from CD44(+) cell to EMT cell drives the metastasis of prostate cancer. Oncotarget. 2015; 6: 1202-16.

25. Li N, Wang C, Wu Y, Liu X, Cao X. Ca(2+)/calmodulin-dependent protein kinase II promotes cell cycle progression by directly activating MEK1 and subsequently modulating p27 phosphorylation. The Journal of biological chemistry. 2009; 284: 3021-7.

26. Mamaeva OA, Kim J, Feng G, McDonald JM. Calcium/calmodulin-dependent kinase II regulates notch-1 signaling in prostate cancer cells. Journal of cellular biochemistry. 2009; 106: 25-32.

27. Jing Y, Han Z, Zhang S, Liu Y, Wei L. Epithelial-Mesenchymal Transition in tumor microenvironment. Cell \& bioscience. 2011; 1: 29.

28. Rokhlin OW, Taghiyev AF, Bayer KU, Bumcrot D, Koteliansk VE, Glover RA, et al. Calcium/calmodulin-dependent kinase II plays an important role in prostate cancer cell survival. Cancer biology \& therapy. 2007; 6: 732-42.

29. Wang C, Li N, Liu X, Zheng Y, Cao X. A novel endogenous human CaMKII inhibitory protein suppresses tumor growth by inducing cell cycle arrest via p27 stabilization. The Journal of biological chemistry. 2008; 283: 11565-74.

30. Wang T, Guo S, Liu Z, Wu L, Li M, Yang J, et al. CAMK2N1 inhibits prostate cancer progression through androgen receptor-dependent signaling. Oncotarget. 2014; 5: 10293-306.

31. Wang T, Liu Z, Guo S, Wu L, Li M, Yang J, et al. The tumor suppressive role of CAMK2N1 in castration-resistant prostate cancer. Oncotarget. 2014; 5: 3611-21.

32. Romanuik TL, Ueda T, Le N, Haile S, Yong TM, Thomson T, et al. Novel biomarkers for prostate cancer including noncoding transcripts. The American journal of pathology. 2009; 175: 2264-76.

33. Biddle A, Liang X, Gammon L, Fazil B, Harper LJ, Emich H, et al. Cancer stem cells in squamous cell carcinoma switch between two distinct phenotypes that are preferentially migratory or proliferative. Cancer research. 2011; 71: 5317-26.

34. Hall CL, Kang S, MacDougald OA, Keller ET. Role of Wnts in prostate cancer bone metastases. Journal of cellular biochemistry. 2006; 97: 661-72.

35. McDonald SL, Silver A. The opposing roles of Wnt-5a in cancer. Br J Cancer. 2009; 101: 209-14

36. Pukrop T, Binder C. The complex pathways of Wnt 5 a in cancer progression. J Mol Med (Berl). 2008; 86: 259-66.

37. Khaja AS, Egevad L, Helczynski L, Wiklund P, Andersson T, Bjartell A. Emphasizing the role of Wnt5a protein expression to predict favorable outcome after radical prostatectomy in patients with low-grade prostate cancer. Cancer Med. 2012; 1: 96-104.

38. Syed Khaja AS HL, Edsjo“ A, Ehrnstro“m R, Lindgren A, et al. Elevated Level of Wnt5a Protein in Localized Prostate Cancer Tissue Is Associated with Better Outcome. PloS one. 2011; 6: e26539.

39. Yamamoto $\mathrm{H}$, Oue $\mathrm{N}$, Sato A, Hasegawa $\mathrm{Y}$, Yamamoto $\mathrm{H}$, Matsubara A, et al. Wnt5a signaling is involved in the aggressiveness of prostate cancer and expression of metalloproteinase. Oncogene. 2010; 29: 2036-46.

40. Thiele S, Rauner M, Goettsch C, Rachner TD, Benad P, Fuessel S, et al. Expression profile of WNT molecules in prostate cancer and its regulation by aminobisphosphonates. Journal of cellular biochemistry. 2011; 112: 1593-600.

41. Sandsmark E, Hansen AF, Selnaes KM, Bertilsson H, Bofin AM, Wright AJ, et al. A novel non-canonical Wnt signature for prostate cancer aggressiveness. Oncotarget. 2017; 8: 9572-86.

42. Ren D, Dai Y, Yang Q, Zhang X, Guo W, Ye L, et al. Wnt5a induces and maintains prostate cancer cells dormancy in bone. J Exp Med. 2019; 216: $428-49$.

43. Thiele S, Zimmer A, Gobel A, Rachner TD, Rother S, Fuessel S, et al. Role of WNT5A receptors FZD5 and RYK in prostate cancer cells. Oncotarget. 2018; 9: 27293-304.

44. Lee GT, Kwon SJ, Kim J, Kwon YS, Lee N, Hong JH, et al. WNT5A induces castration-resistant prostate cancer via CCL2 and tumour-infiltrating macrophages. Br J Cancer. 2018; 118: 670-8.

45. Tamura $\mathrm{K}$, Furihata $\mathrm{M}$, Satake $\mathrm{H}$, Hashida $\mathrm{H}$, Kawada $\mathrm{C}$, Osakabe $\mathrm{H}$, et al. SHISA2 enhances the aggressive phenotype in prostate cancer through the regulation of WNT5A expression. Oncol Lett. 2017; 14: 6650-8. 